\title{
Light Higgs Discovery Potential with ATLAS, Measurements of Couplings and I mpact on Model Discrimination
}

\author{
J unichi TANAKA \\ I CEPP, Univ. of TOKYO
}

On behalf of the ATLAS Collaboration 


\section{Outline}

- LHC Schedule

- ATLAS Detector

- Motivation

1. Light Higgs Discovery Potential

- Production cross section and branching ratio of SM Higgs

- $\mathrm{H}->\gamma \gamma, \mathrm{H}->\tau \tau$ and $\mathrm{H}->\mathrm{WW}$

- Discovery potential

2. Measurement of Couplings and Model Discrimination

- Summary 


\section{LHC Schedule}

LHC is composed of 1232

superconducting dipole magnet.

- Length $=14 \mathrm{~m}$

- Provide B=8.3Tesla working at 1.9

Kelvin

- $90 \%$ of them were assembled.

- $40 \%$ of them were installed in the

LHC tunnel.

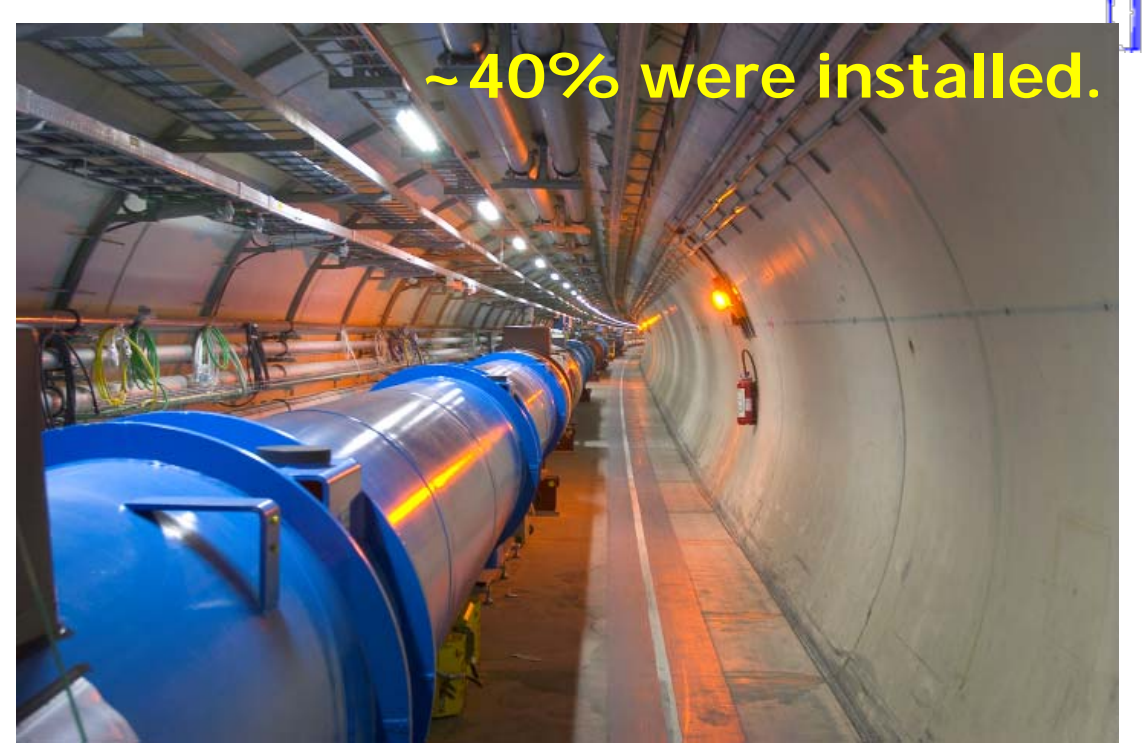

Two general-purpose pp experiments:

ATLAS and CMS

- April, 2007 : cosmic and single beam

with lower energy Tevatron?

- Summer, 2007 : physics run $->\mathrm{L}=\sim 0.1 \mathrm{fb}^{-1}$ in 2007 Calibration

- $\mathrm{L}=\sim \mathrm{a}$ few $\mathrm{fb}^{-1}$ in 2008 SUSY?, Higgs? with 14TeV

- $\mathrm{L}=\sim 10 \mathrm{fb}^{-1}$ in 2009 , then increase the luminosity to the design.

\section{Higgs}




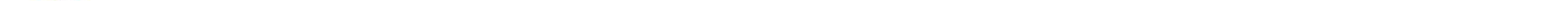




\section{Motivation}

- Discovery of Higgs boson is one of the most important purposes of LHC.

- At the present we, ATLAS, focus on studies with the early physics data.

- How to calibrate/estimate/evaluate what we need using data of 0.1$1 . \mathrm{Ofb}^{-1}$.

- After the discovery,

- We have to identify the Higgs Boson. Is it SM Higgs or something else?

-> We measure mass, coupling, spin, CP and so on.

In this talk,

1. Discovery Potential of Light Higgs at ATLAS

- $\mathrm{H}->\gamma \gamma, \mathrm{H}->\tau \tau$ and $\mathrm{H}->\mathrm{WW}$

2. Measurement of Coupling and Discrimination between SM and MSSM 


\section{Discovery Potential of Light Higgs at ATLAS}

- The present experimental data prefers light Higgs $\left(\mathrm{M}_{\mathrm{H}}<\sim 200 \mathrm{GeV}\right)$ to heavy.

- SUSY predicts the existence of a light Higgs, whose mass is less than $\sim 130 \mathrm{GeV}$.

- Experimental signature of MSSM Lightest CP-even Neutral Higgs is similar to SM Higgs.

- Signal rate is different with the SM case due to

LEP EWWG, March 06 couplings.

-> Can use this characteristics in order to distinguish MSSM from SM (see the next topics).

- Studies of SM Higgs are shown here.

- $\mathrm{M}_{\mathrm{H}}<\sim 140 \mathrm{GeV}: \mathrm{H}->\gamma \gamma$ and $\mathrm{H}->\tau \tau$

$-\mathrm{M}_{\mathrm{H}}>\sim 130 \mathrm{GeV}: \mathrm{H}->\mathrm{WW}$ 


\section{$\mathbf{H}->\gamma \gamma$}

- Study of inclusive analysis, mainly gg- $>\mathrm{H}->\gamma \gamma$, has been continued.

- Huge BG -> S/B 3\%

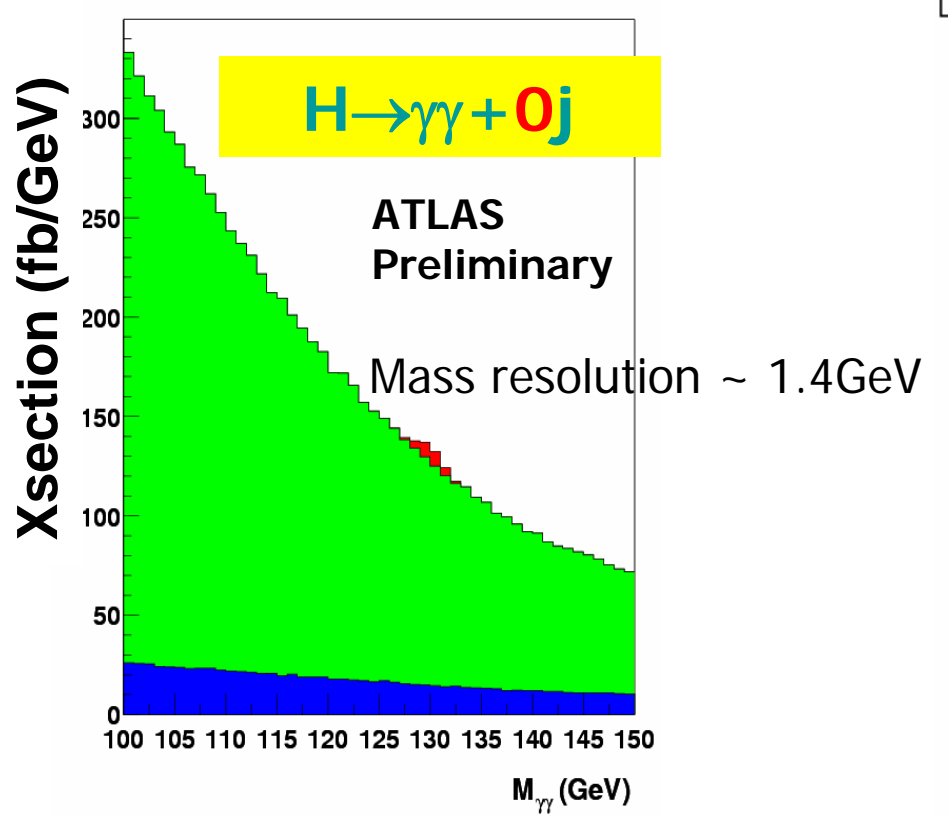

R ... Signal

G ... I rreducible BG

B ... Reducible BG
Signal significance: updated DC1 analysis

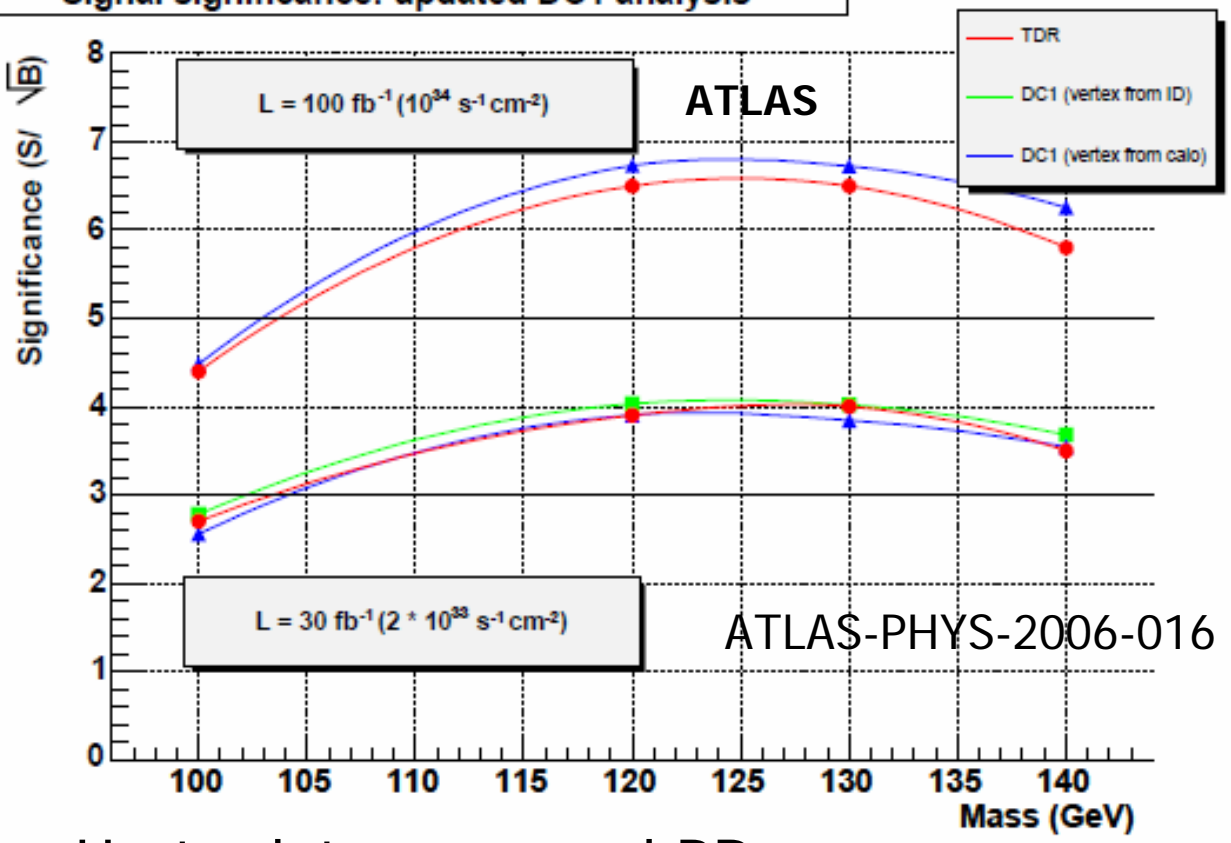

Up-to-date $x$-sec and BR Photon ID etc.

-> Almost same result as our TDR result 
- VBF and $\mathrm{H}+1$ jet analysis

- S/B is much better than inclusive analysis.

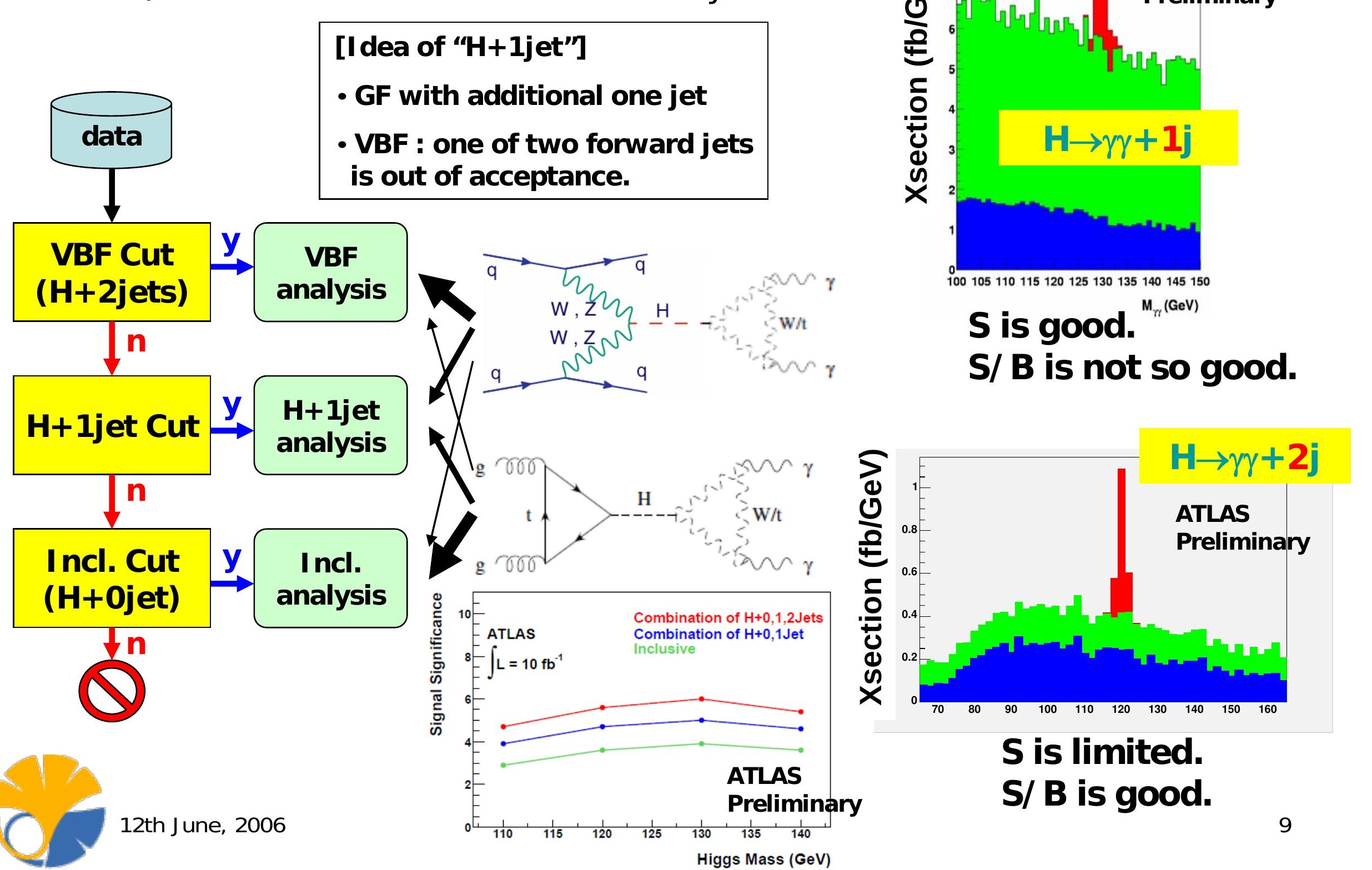




\section{$\mathbf{H}->\gamma \gamma$}

[Working on]

- Study of additional jet(s)

- Uncertainty from such a jet in the analysis of $\mathrm{H}+1$ jet and VBF

- Understand it by using Alpgen, MC@NLO and so on.

- Photon calibration : energy scale and resolution

- Photon ID : Fake photons from jets

- When $\pi^{0}$ in a jet carries most of the jet energy, this $\pi^{0}$ can be misidentified as a photon.

-> A jet rejection factor of $\sim 5000$ for $\mathrm{Pt}>25 \mathrm{GeV}$ is obtained for a photon ID efficiency $\sim 80 \%$.

- Conversion (recovery)

- $\quad \sim 40 \%$ of gamma is converted. Some of them cannot be identified as gamma.

- Photon angle correction : help of calorimeter pointing 


\section{VBF $\mathbf{H}->\tau \tau$}

- We can observe a Higgs mass peak on the right side of the Drell-Yan peak.

- We can reconstruct $M(\tau \tau)$, which corresponds to Higgs mass, using missing Et with the collinear assumption.

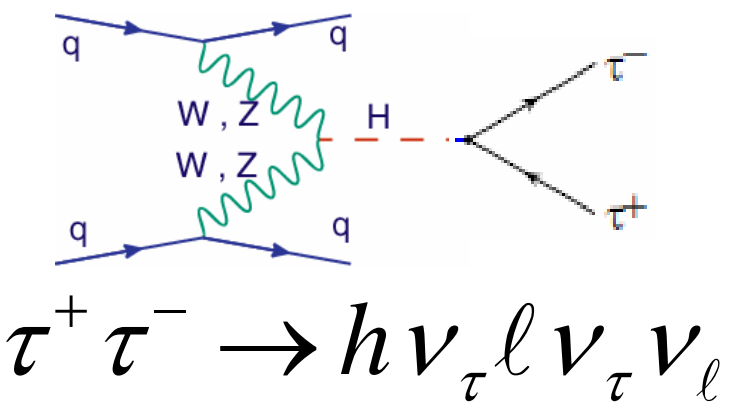

Eur.Phys.J .,C32(2004) 19

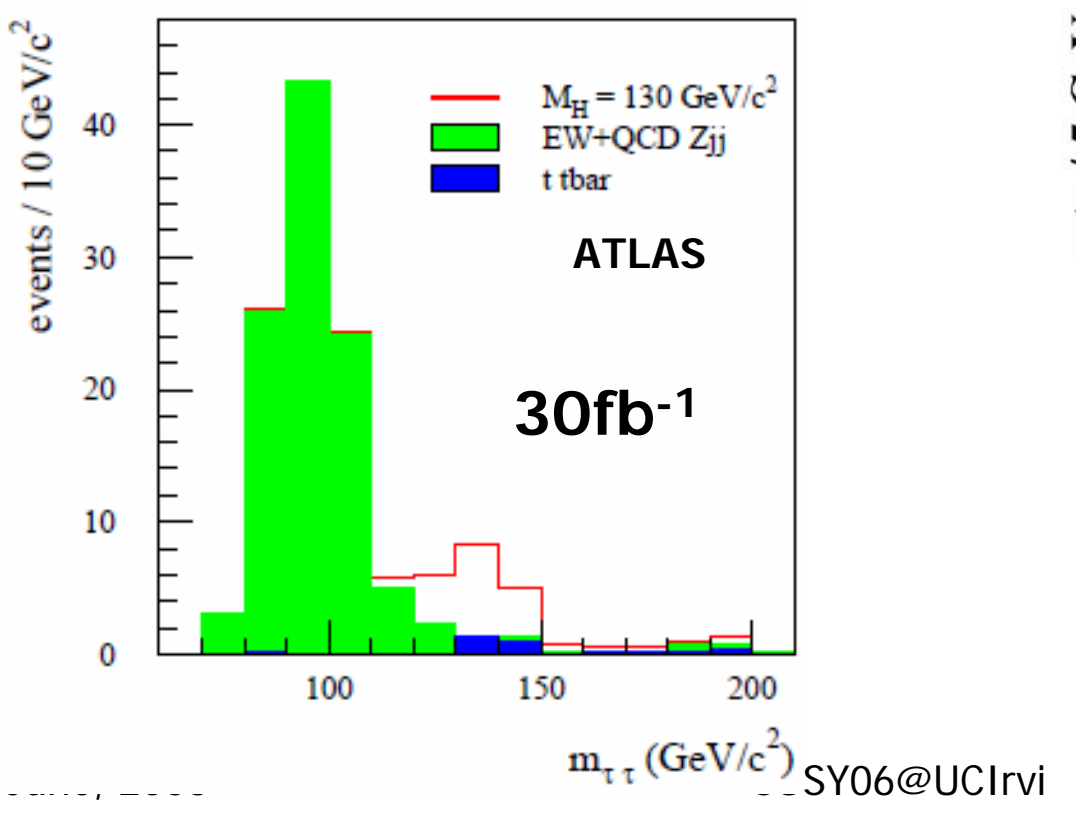

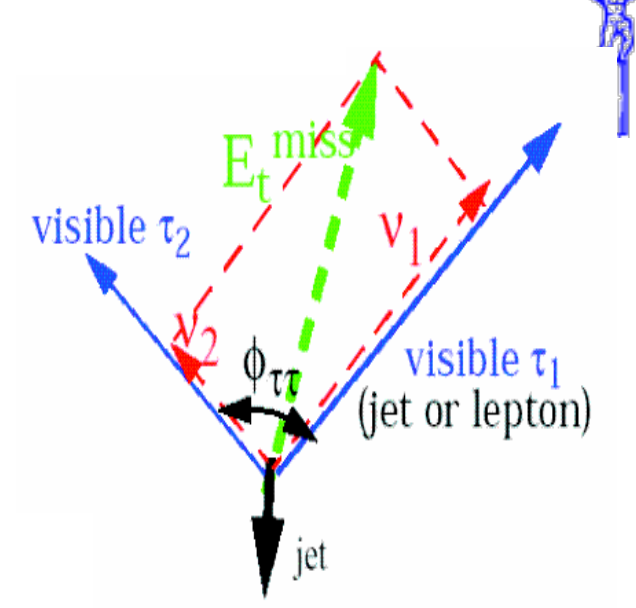

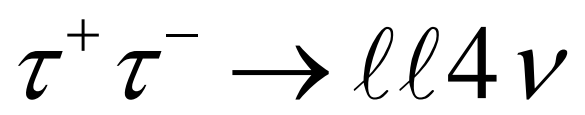

Eur.Phys.J., C32(2004) 19

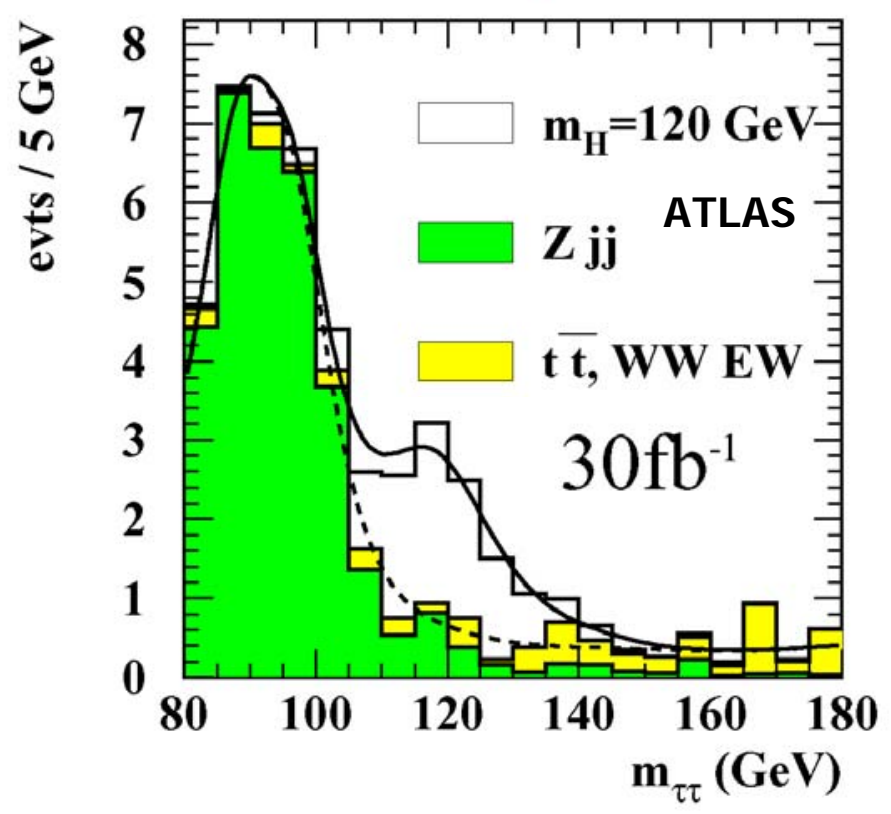


[Working on]

- Study of $3^{\text {rd }}$ jet

- Rapidity gap is a crucial signature of VBF.

- Understand this uncertainty using several generators.

- Tau identification

- Reject QCD backgrounds and keep/increase signal events

- We have developed a "track based" tau reconstruction tool.

- Mass reconstruction

- Resolution of Missing Et

- Background estimation using real data

- A tail from the Drell-Yan peak is in the signal region.

- Estimation of this tail using real data is a key issue.

-> Under study. 


\section{VBF $\mathbf{H}->\mathbf{W W}->\|_{l v v}$}

- $\mathrm{H}->\mathrm{WW}$ dominates when Higgs mass relatively becomes large.

$\left(\mathrm{M}_{\mathrm{H}}>\sim 130 \mathrm{GeV}\right)$

- Cannot observe a Higgs peak.

-> Check transverse mass.

$$
M_{T}=\sqrt{2 P_{T}(\ell \ell) E_{T}^{m i s s}(1-\cos \Delta \phi)}
$$

- S/B is good.

[working on]

- Background estimation is a key issue.

- Use real data

-> Under study
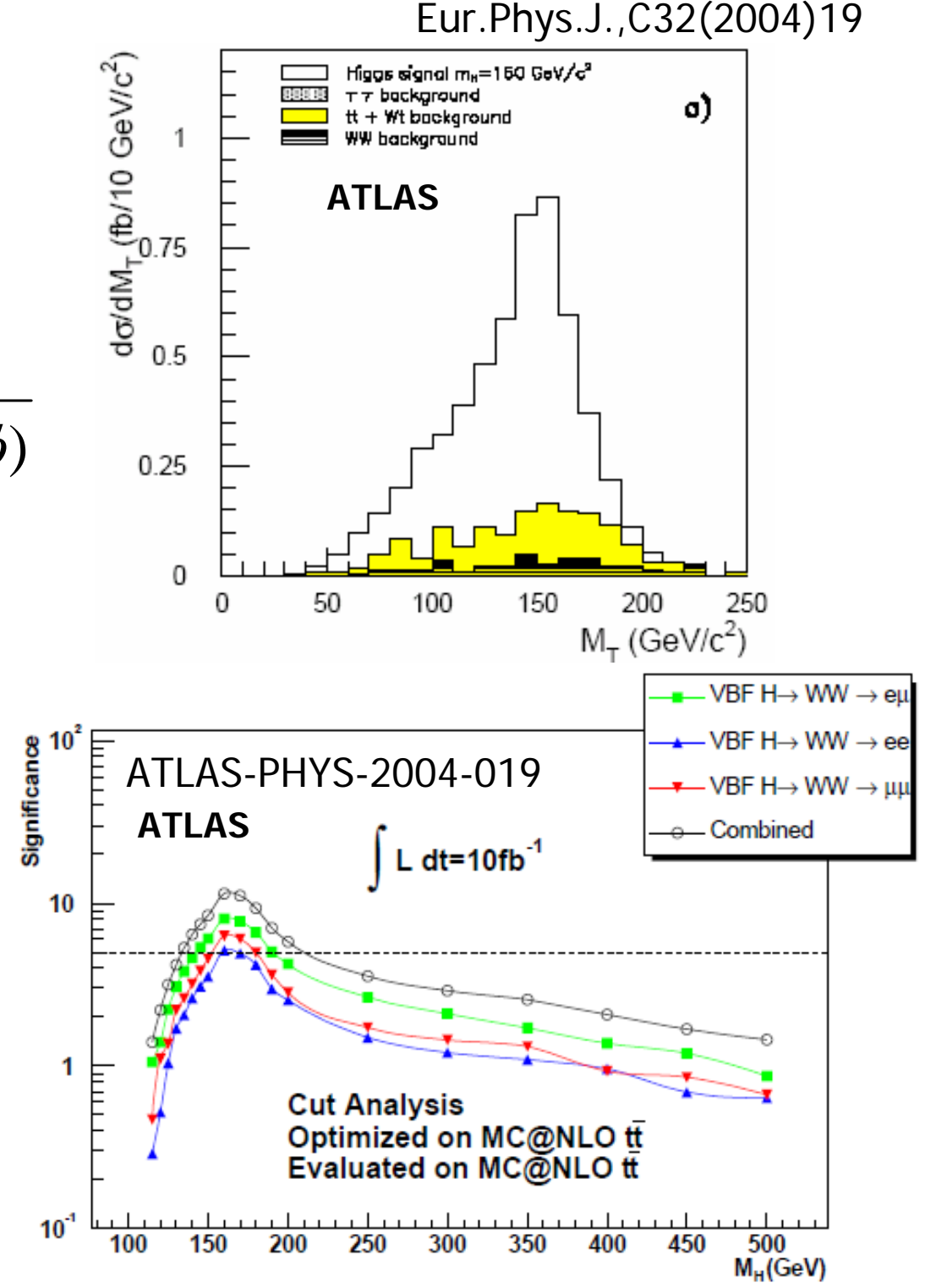


\section{Discovery Potential of SM Higgs}

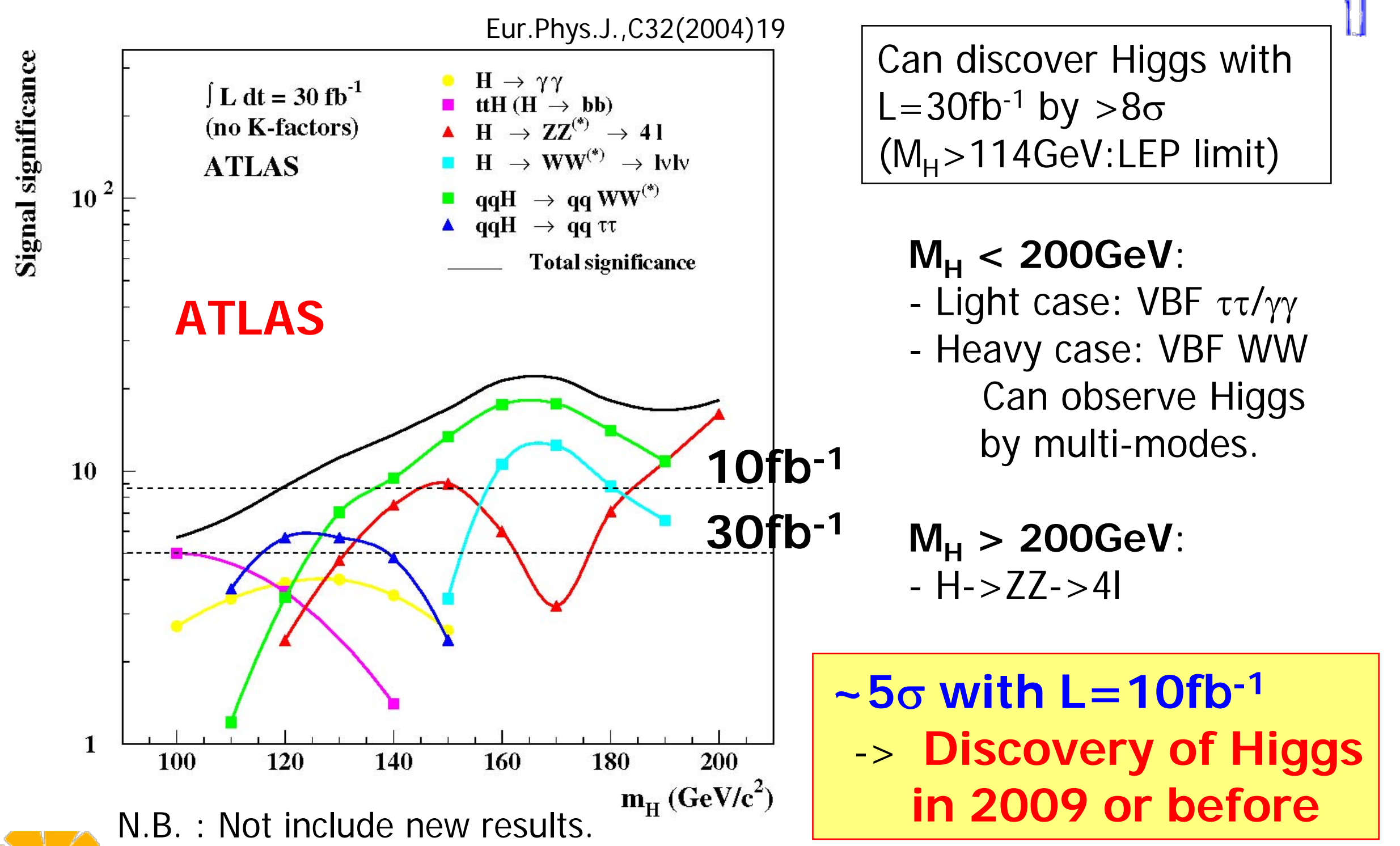




\section{Measurement of Coupling and Discrimination between SM and MSSM}

- Yukawa coupling

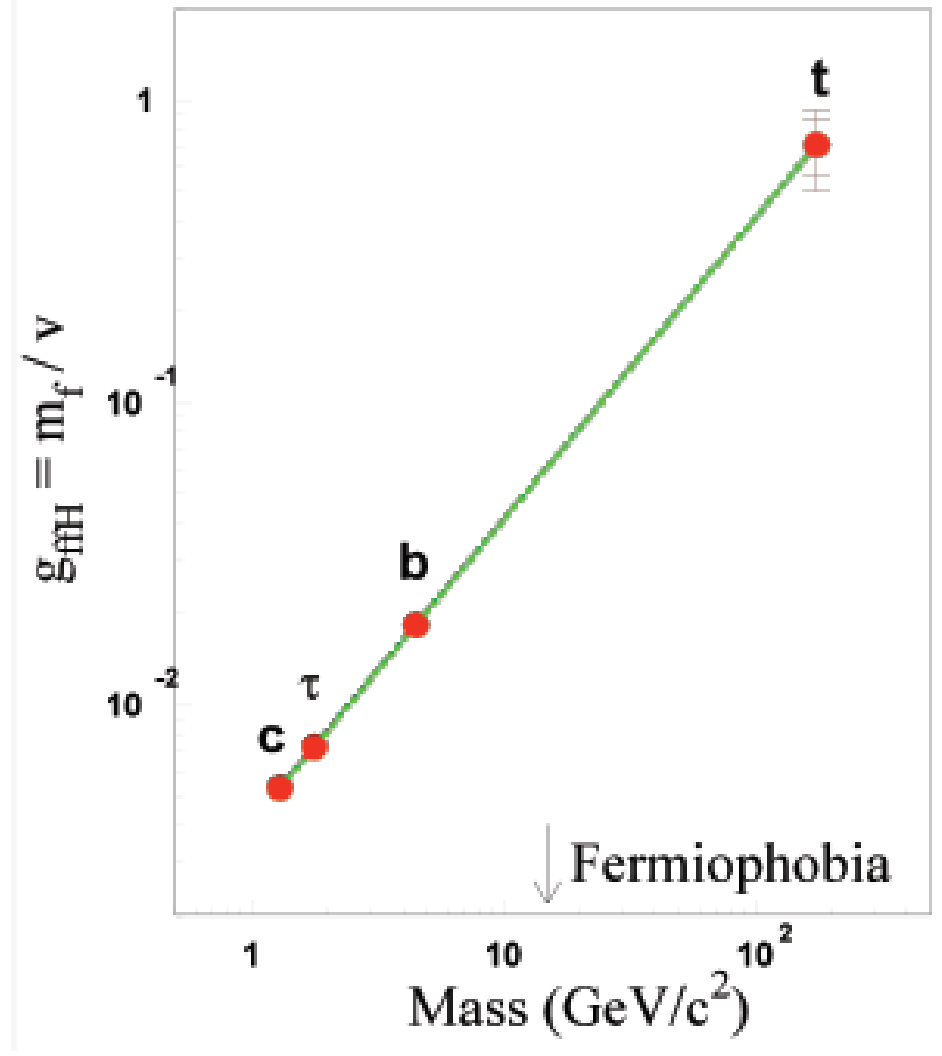

-> Prove the origin of mass

- Check the linearity of Yukawa coupling as a function of fermion mass. 


\section{Ratio of Couplings}

- In the low mass region, we can measure event rates of Higgs with multi modes. So we can extract coupling information, particularly, determination of ratios is possible with a model-independent way.
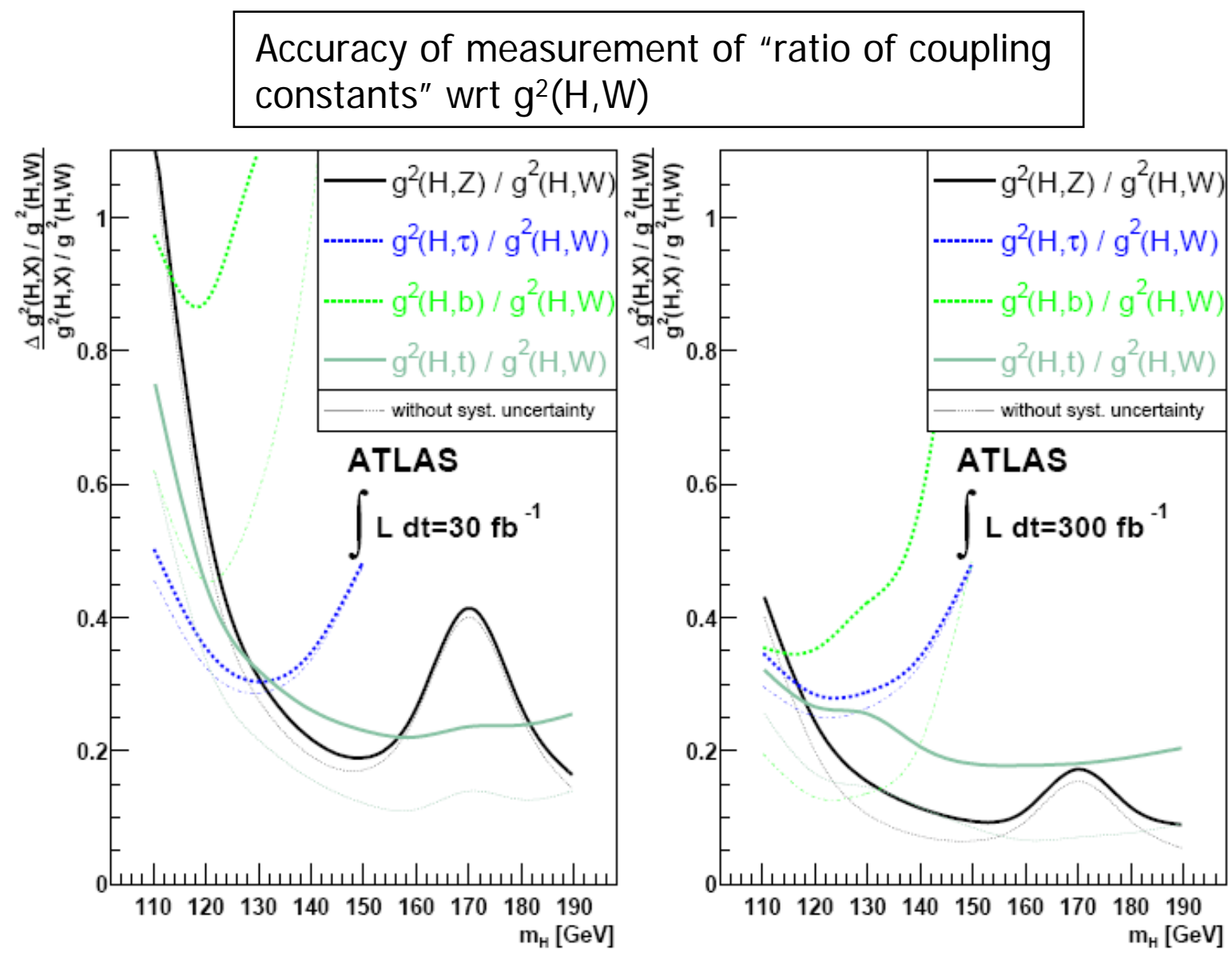


\section{Absolute Coupling Determination}

- Need a help of theory to obtain the absolute values of couplings.

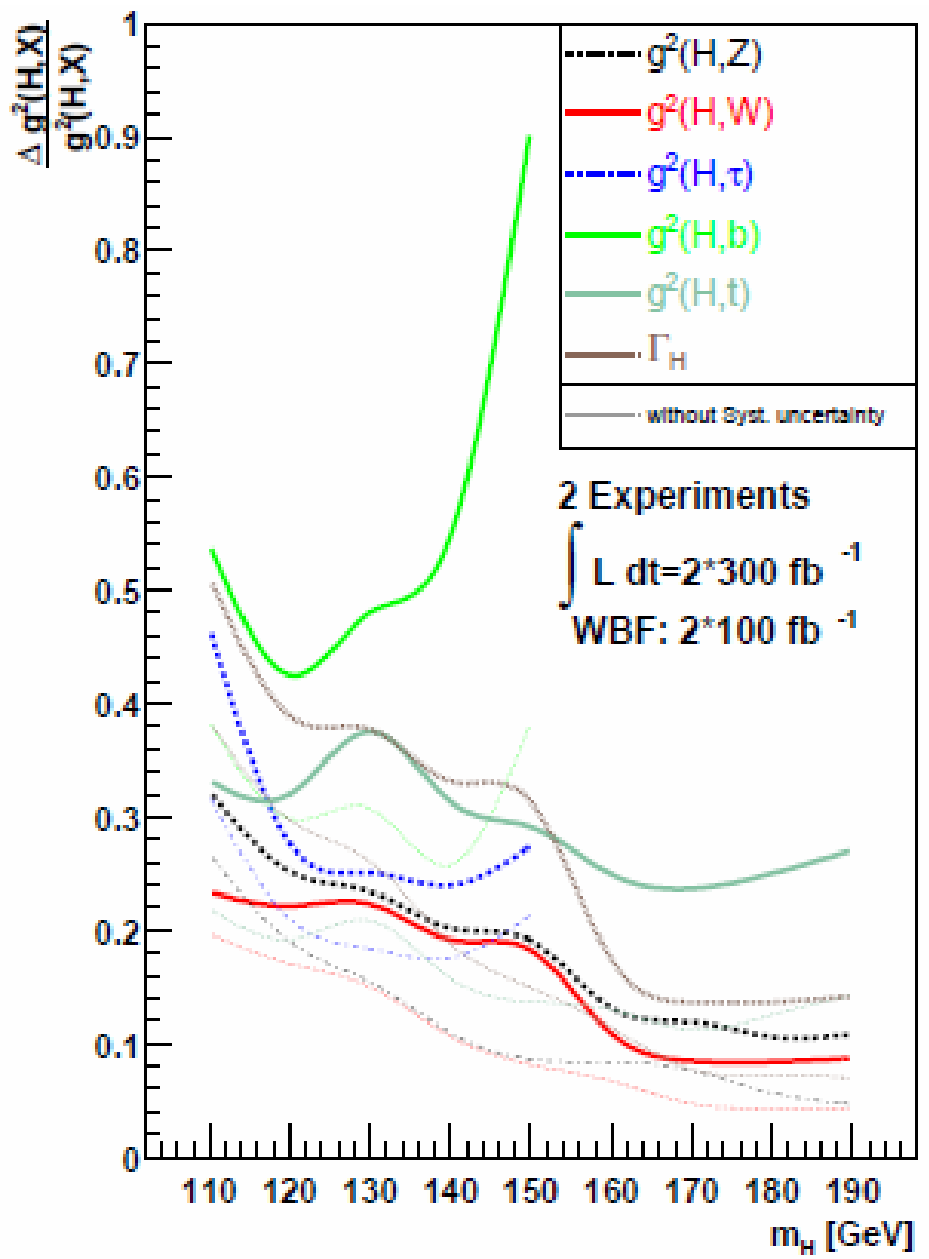

Assumption [hep-ph/0407190] :

- $\mathrm{HW}(\mathrm{V}=\mathrm{W}, \mathrm{Z})$ couplings cannot be larger than the SM case, namely,

- $g^{2}(H, W)<g_{S M}^{2}(H, W)$

- $g^{2}(H, Z)<g^{2}{ }_{S M}(H, Z)$

This constraint is valid in generic multi-Higgs-doublet models. (eg. MSSM)

With $2 \times 300 \mathrm{fb}^{-1}$ :

- Yukawa

- $g_{\mathrm{t}}^{2}: 25-40 \%$

- $g_{b}^{2}: 45-90 \%$

- $g_{\tau}^{2}: 25-50 \%$

- Gauge Boson

- $g_{z}^{2}: 10-30 \%$

- $\mathrm{g}_{\mathrm{w}}^{2}: 10-25 \%$

- Total Width : 15-50\% 


\section{Discrimination between SM and MSSM}

- Use only event rates of Higgs to distinguish the SM from a specific MSSM scenario. [hep-ph/0407190 -> no-mixing scenario]
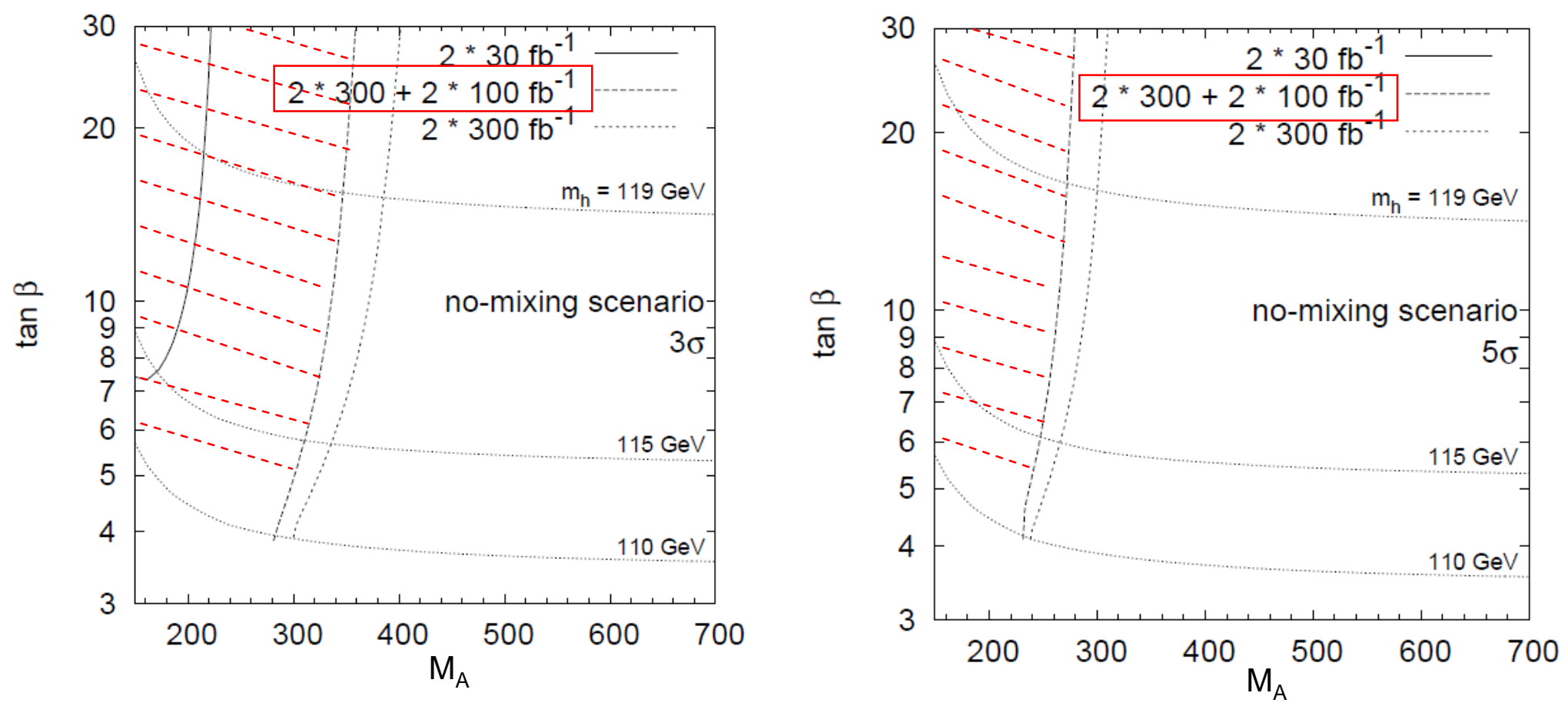

- 3б: $M_{A}<350 G e V, 5 \sigma: M_{A}<250 G e V$.

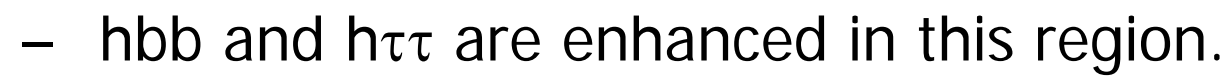

- We also expect observations of multi Higgs. 


\section{Summary}

- Present experimental data prefers Light Higgs $\left(M_{H}<200 G e V\right)$ to Heavy.

- Light Higgs is interesting from a point of view of both discovery and parameter determination.

- Thanks to observations with multi modes.

- ATLAS can discover a Higgs boson with $10 \mathrm{fb}^{-1}$ or less.

- We are working very hard to establish how to calibrate/estimate/evaluate what we need (if possible) using the real data.

- In the low mass region, couplings and the total width can be determined with the accuracy of a several $10 \%$ using $(2 \times) 300 \mathrm{fb}^{-1}$ of data at LHC.

- Real (14TeV) physics run will start in 2008. 


\section{Backup}




\section{SM Higgs mass}

- From the precision measurements:

$$
m_{\mathrm{H}}=91_{-32}^{+45} \mathrm{GeV}
$$

Including theory uncertainty:

$$
m_{H}<186(95 \% C L)
$$

Direct search of LEP:

$$
m_{H}>114 \mathrm{GeV}(95 \% \mathrm{CL})
$$

Renormalize probability for $m_{\mathrm{H}}>114 \mathrm{GeV}$ to $100 \%$ :

$$
\mathrm{m}_{\mathrm{H}}<219 \mathrm{GeV}(95 \% \mathrm{CL})
$$

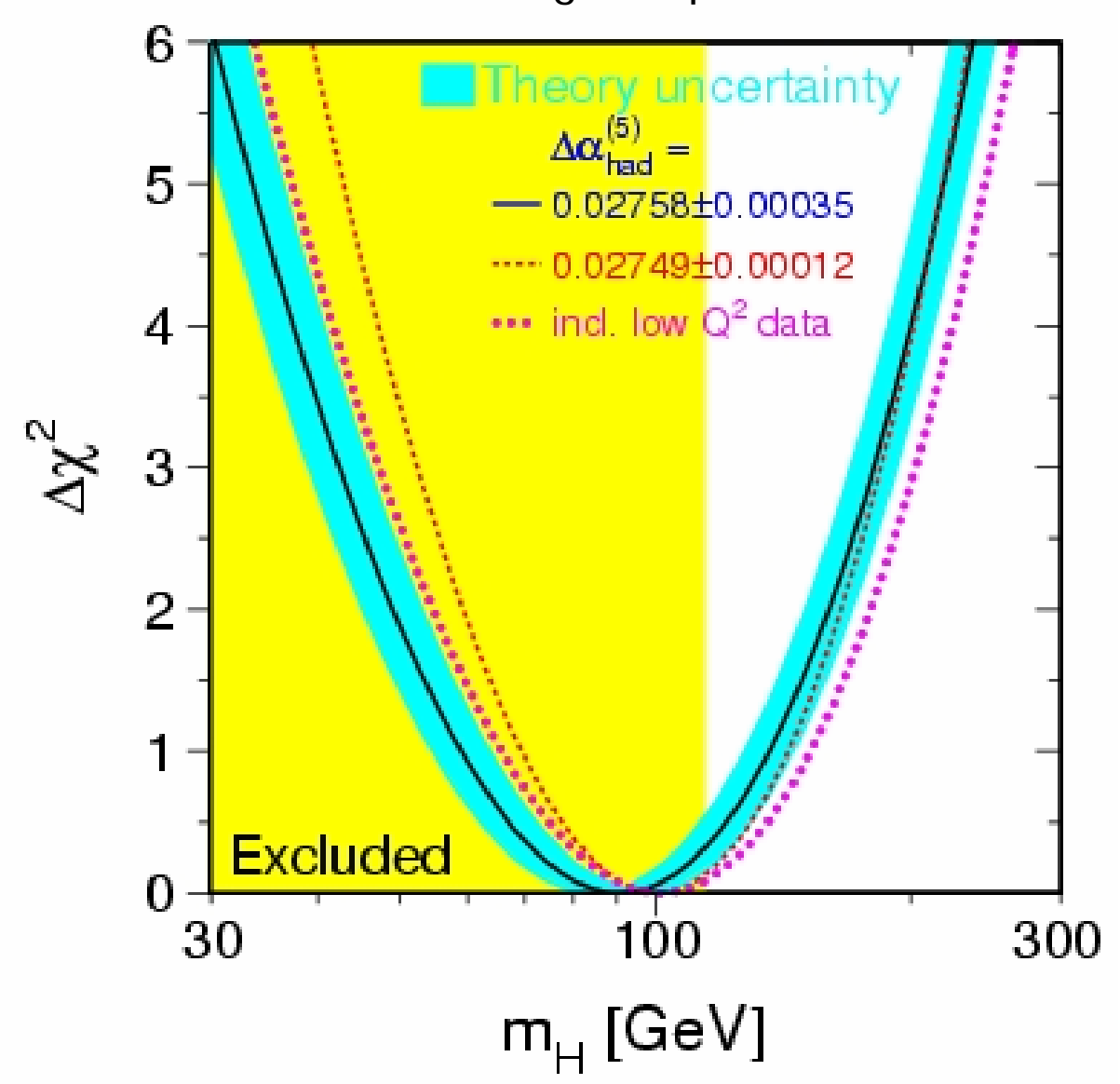

We put the SM Higgs into a corner. LHC can discover the SM Higgs. 


\section{Summary of Studies for SM Higgs}

\section{Combination of production and decay}

\begin{tabular}{|c|c|c|c|}
\hline Production & Decay & Mass region a & d purpose \\
\hline \multirow{3}{*}{ Gluon Fusion } & $\mathbf{H}->\gamma \gamma$ & 110-140GeV & Discovery, Mass \\
\hline & $H->Z Z->4 I$ & $140-1000 \mathrm{GeV}$ & Discovery, Mass, spin, coupling \\
\hline & $\mathrm{H}->\mathrm{WW}$ & 130-170 GeV & Discovery \\
\hline \multirow{4}{*}{$\begin{array}{l}\text { Vector Boson } \\
\text { Fusion }\end{array}$} & $\mathbf{H}->\tau \tau$ & 110-140GeV & Discovery, Mass, coupling \\
\hline & $H->W W$ & 130-200GeV & Discovery, W coupling \\
\hline & $\mathbf{H}->\gamma \gamma$ & $110-140 \mathrm{GeV}$ & Discovery, Mass \\
\hline & $\mathrm{H}->\mathrm{bb}$ & $110-140 \mathrm{GeV}$ & $\begin{array}{l}\text { Yb coupling (need study of } \\
\text { trigger) }\end{array}$ \\
\hline \multirow{3}{*}{$\mathrm{ttH}$} & $\mathrm{H}->\mathrm{bb}$ & 110-130GeV & \multirow{3}{*}{ Yt coupling } \\
\hline & $\mathrm{H}->\tau \tau$ & $110-130 \mathrm{GeV}$ & \\
\hline & $\mathrm{H}->\mathrm{WW}$ & 130-180GeV & \\
\hline WH & $\mathrm{H} \rightarrow \mathrm{WW}$ & 140-170GeV & Discovery, W coupling \\
\hline
\end{tabular}




\section{MSSM Higgs Sensitivity for $5 \sigma$-discovery}

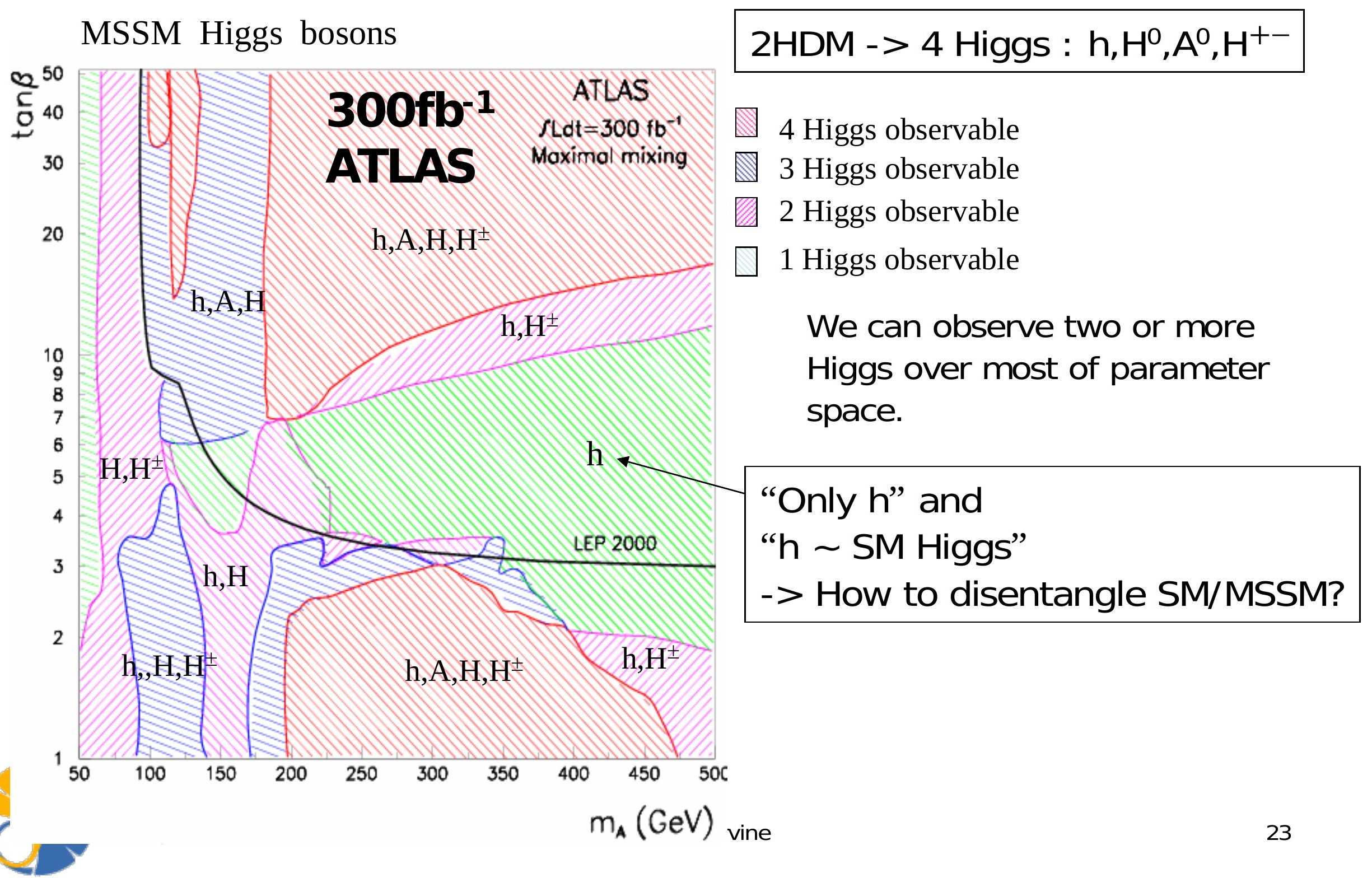

